\title{
The effects of dynamic ionospheric outflow on the ring current
}

\author{
D. T. Welling, ${ }^{1}$ V. K. Jordanova, ${ }^{1}$ S. G. Zaharia, ${ }^{1}$ A. Glocer, ${ }^{2}$ and G. Toth ${ }^{3}$ \\ Received 5 May 2010; revised 6 December 2010; accepted 21 December 2010; published 26 February 2011.
}

[1] The importance of ionospheric $\mathrm{O}^{+}$on the development of the storm time ring current is recognized but not well understood. The addition of this outflow in global MHD models has the potential to change the magnetic field configuration, particle densities and temperatures, and the convection electric field. This makes including heavy ion outflow in ring current simulations difficult, as this addition cannot be easily decoupled from a host of other changes. This study attempts to overcome this problem by using three coupled models, PWOM, RIM, and BATS-R-US, to drive a ring current model, RAM-SCB. The differences in drivers when outflow is included and is not included are compared to see how outflow changes ring current input. It is found that including this outflow reduces the convection electric field, lowers the plasma sheet number density and temperature, and increases the complexity of the plasma sheet ion composition both temporally and spatially. These changes cause an overall reduction in ring current energy density. Further simulations that attempt to isolate these effects find that the most important change in terms of ring current development is the drop in convection electric field. Local time dependencies of $\mathrm{O}^{+}$injections are found to be nontrivial as well. Capturing all of these effects requires a whole system, first-principles approach.

Citation: Welling, D. T., V. K. Jordanova, S. G. Zaharia, A. Glocer, and G. Toth (2011), The effects of dynamic ionospheric outflow on the ring current, J. Geophys. Res., 116, A00J19, doi:10.1029/2010JA015642.

\section{Introduction}

[2] Since the discovery of oxygen ions in the magnetosphere [Shelley et al., 1974], it has become widely recognized that the ionospheric outflow of heavy ions plays an important role in magnetospheric dynamics and ring current development during geomagnetic storms. Numerous studies have confirmed the ionosphere's contributions to magnetospheric plasma populations [e.g., Lennartsson and Shelley, 1986; Nosé et al., 2003; Denton et al., 2005]. Sharp et al. [1985] examined data from the International Sun-Earth Explorer (ISEE) and Spacecraft Charging AT High Altitude (SCATHA) spacecrafts and determined that the ionosphere was an important or dominant source of plasma to the inner magnetosphere. Daglis et al. [1999], in a review of the ring current, summarize ring current composition measurements to show how $\mathrm{O}^{+}$increases during active periods. Chappell et al. [1987] suggested that the ionosphere could adequately and independently provide ample plasma to the magnetosphere to agree with observations. The more recent work by Huddleston et al. [2005] supported this hypothesis by using measurements from the Polar satellite to seed a particle tracing simulation.

[3] Because of the importance of the heavy ion contribution, numerical studies of the ring current have attempted

\footnotetext{
${ }^{1}$ Los Alamos National Laboratory, Los Alamos, New Mexico, USA.

${ }^{2}$ NASA Goddard Space Flight Center, Greenbelt, Maryland, USA.

${ }^{3}$ Department of Atmospheric, Oceanic, and Space Sciences, University of Michigan, Ann Arbor, Michigan, USA.
}

Copyright 2011 by the American Geophysical Union. 0148-0227/11/2010JA015642 to include this source using various methods. This remains a challenging task for a number of reasons. The mechanisms responsible for driving outflow as well as their dependence on solar wind and magnetospheric conditions are not fully understood. The transport of these ions to the plasma sheet and inner magnetosphere is equally complicated and subject to continual investigation. Hence, obtaining a realistic distribution of outflowing ions, accelerating this distribution into the magnetosphere, then properly advecting it to the ring current creates a multitiered problem. Attempting to avoid these complexities by incorporating measurements into simulations fails to fully alleviate the problem, as observations are sparse, both spatially and temporally.

[4] Jordanova et al. [2006] combined measurements from the Magnetospheric Plasma Analyzer (MPA) instrument aboard the Los Alamos National Laboratory geosynchronous satellites [McComas et al., 1993] with the empirical relationship between the geomagnetic $\mathrm{Kp}$ index and solar F10.7 flux as formulated by Young et al. [1982] to create boundary conditions for the Ring current Atmosphere interactions Model [Jordanova et al., 1996, 1997]. This approach has its basis in observations, maintains local time variation in fluxes, and provides temporally dynamic $\mathrm{O}^{+}$composition. However, it ties the two species together such that the $\mathrm{O}^{+}$ density is always a spatially constant ratio of the $\mathrm{H}^{+}$density. Fok et al. [2006] traced $\mathrm{O}^{+}$from ionospheric sources through the fields generated by the Lyon, Fedder, and Mobarry (LFM) 3-D magnetohydrodynamic (MHD) code [Lyon et al., 2004] to provide fluxes at the outer boundary of the Comprehensive Ring Current Model [Fok et al., 2001]. $\mathrm{O}^{+}$particles were initialized using the MHD values near the inner boundary 
and leveraging relationships formulated by Strangeway et al. [2005] and Zheng et al. [2005]. While this methodology provides causal outflow and includes the complexities associated with transport to the plasma sheet and ring current, the tracing of $\mathrm{O}^{+}$through the MHD results does not self consistently affect the magnetosphere-ionosphere system.

[5] Recently, global MHD simulations have added causally driven outflow capabilities. Winglee [1998] added gravitationally bound $\mathrm{O}^{+}$to multifluid MHD simulations that could be accelerated into the domain through pressure gradients or entrifugal acceleration. This led to the discovery that including such outflow could significantly lower the Cross Polar Cap Potential (CPCP) [Winglee et al., 2002]. Later, Gagne [2005] added to the LFM model outflow dictated by the Strangeway et al. [2005] relation. More recently, Glocer et al. [2009a] created a first principles based outflow model and incorporated it into the Space Weather Modeling Framework [Tóth et al., 2005] to provide dynamic outflow in the Block Adaptive Tree Solar wind Roe-type Upwind Scheme (BATS-R-US) global MHD code. This resulted in an impact on the CPCP similar to that found by Winglee et al. [2002]. Brambles et al. [2010] and Wiltberger et al. [2010] have expanded on the work with the LFM model as performed by Gagne [2005], experimenting with outflow fluxes of different velocities and densities. They have found that dense, slow outflow populations impact the magnetosphere in ways similar to those found by Winglee et al. [2002] and Glocer et al. [2009a], but faster flows have limited implications.

[6] These new capabilities allow for an expanded investigation of outflow and its importance to the ring current. How does the delivery of heavy ions change throughout a geomagnetic storm? How does this outflow affect the evolution of the ring current? Do the added complexities found in the first-principles-based models have important impacts on the inner magnetosphere?

[7] This study is the first to use a complete first principles based modeling approach, from outflow to inner magnetosphere, to fully investigate the impacts of outflow on storm time ring current development. A multispecies MHD approach is used to deliver heavy and light ions from the ionosphere to the ring current to preserve the composition set by the outflow. This simulation is compared to a similar case that does not include outflow in order to examine the differences between the two and assess the impacts of including this outflow on the whole system.

\section{Methodology}

[8] Three coupled codes are used to simulate outflow, ionospheric electrodynamics, and the global magnetosphere: the Polar Wind Outflow Model (PWOM), the Ridley Ionosphere Model (RIM), and the Block Adaptive Tree Solar wind Roe-type Upwind Scheme magnetohydrodynamic (MHD) model (described below). These codes are coupled through the Space Weather Modeling Framework (SWMF) [Tóth et al., 2005], a flexible tool for coupling and synchronizing many different space weather codes. Working together, these models provide plasma and magnetic field boundary conditions as well as convection electric field for the ring current model, the Ring current Atmosphere interactions Model with Self-Consistent Magnetic Field (RAM-SCB).
The relationship between these models is summarized in Figure 1.

[9] The PWOM [Glocer et al., 2007, 2009a] models the dynamics of $\mathrm{O}^{+}, \mathrm{He}^{+}, \mathrm{H}^{+}$, and electrons in the "gap region", an area between where most ionospheric models stop (around $1000 \mathrm{~km}$ ) and most magnetospheric models begin (2 to 3 Earth Radii, $R_{E}$ ). Vertical transport of the ion species is obtained by solving the gyrotropic transport equations [Gombosi and Nagy, 1989] along a particular magnetic field line. Electron density is obtained by assuming charge neutrality; electron velocity is obtained using electric current density (received from RIM) and assuming steady state electron velocity. Horizontal motion is generated by allowing the field lines to electromagnetically drift through the domain as driven by the convection electric field, again obtained from RIM. The resulting outflow flux from PWOM is used to set the inner boundary mass and radial velocity of the BATS-R-US model. This coupling is described in detail by Glocer et al. [2009a].

[10] This work includes a new improvement to how PWOM calculates electron temperature. In the transition region between the ionosphere and magnetosphere, this temperature is strongly dependent on the topside electron heat flux. In the past, PWOM utilized an average value found by Bekerat et al. [2007] to be useful in predicting DMSP plasma density measurements. The use of an average value of the heat flux was a sufficient starting point, but did not account for the strong variation present at high latitudes. The new calculation in PWOM draws inspiration from the example of Schunk et al. [1986], who demonstrated that a realistic determination of the electron temperature can be obtained by using a reasonable distribution of the topside electron heat flux. Specifically, they note three regions where the heat flux should be elevated: (1) the auroral regions, which are connected to the hottest part of the magnetosphere, (2) the sunlit regions, due to warm photoelectrons, and (3) the cusp, due to enhanced soft precipitation.

[11] PWOM now defines the topside heat flux in a similar manner. The solar zenith angle is used to scale the heat flux associated with photoelectrons:

$$
H F_{\text {sun }}=H F_{\text {sun } 0} \cos (\Xi)
$$

The precipitation calculated by the MHD code is used to scale the heat flux associated with the aurora:

$$
H F_{\text {Aurora }}=H F_{\text {Aurora } 0} \frac{e F l u x}{\text { eFlux } x_{0}}
$$

$H F_{\text {sun }}$ and $H F_{\text {Aurora }}$ refer to the topside electron heat flux from photoelectrons, and auroral precipitation. $\Xi$ is the solar zenith angle which is calculated at each grid point. eFlux is the precipitating electron flux calculated between the magnetosphere model and ionosphere electrodynamics model. $H F_{\text {sun } 0}, H F_{\text {Aurora } 0}$, and eflux 0 are "quiet time" reference values that have been chosen to give temperature values consistent with observations. The cusp associated heat flux is set in the same way as the auroral flux. A minimum value in the polar cap is also specified to represent polar rain. That quantity is set to ensure that we get an $\mathrm{H}^{+} / \mathrm{O}^{+}$crossover altitude consistent with data, approximately $4000 \mathrm{~km}$ [Yau et al., 2007]. Figure 2 illustrates a typical result of this calculation. It shows the 


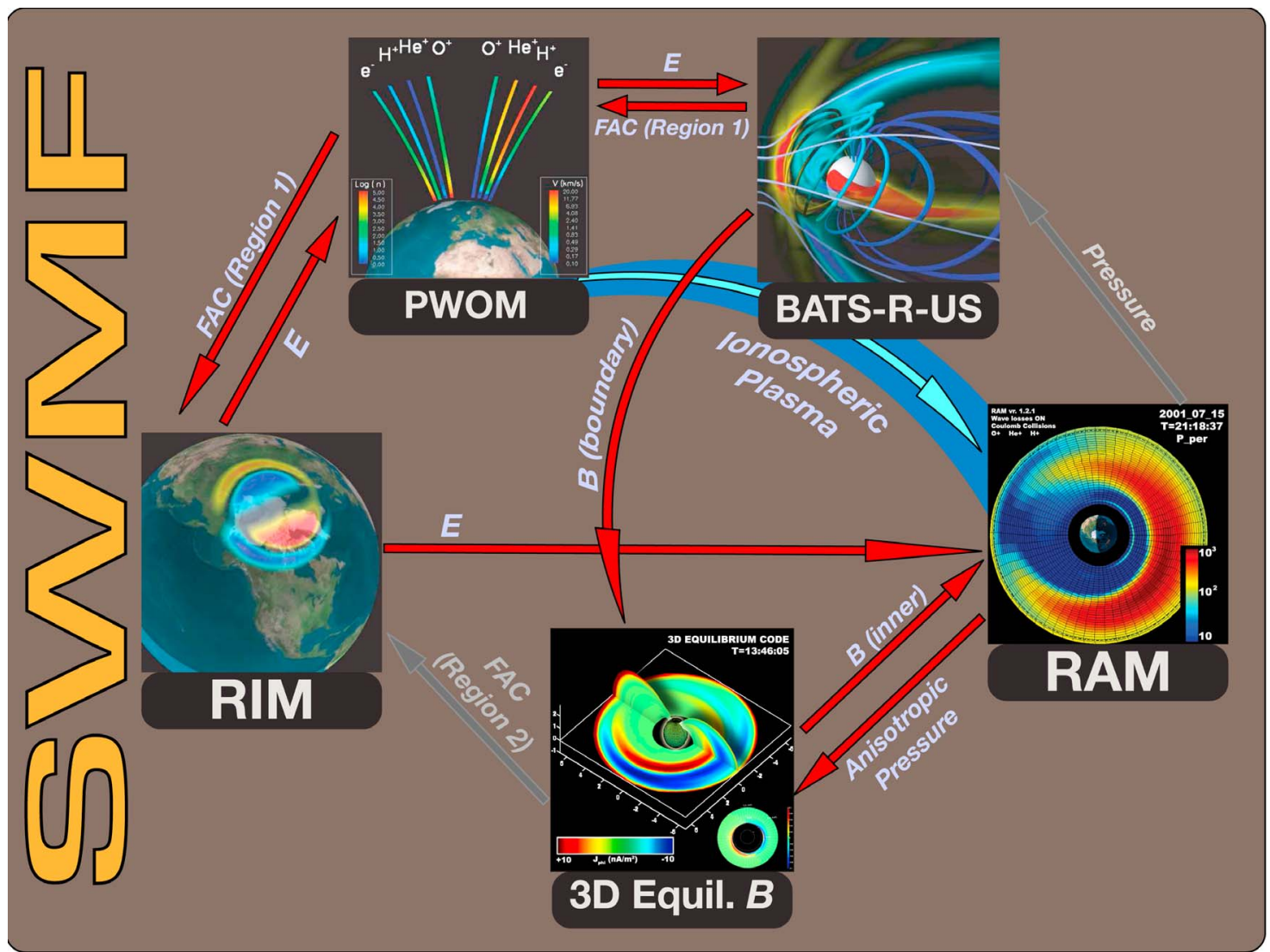

Figure 1. A diagram summarizing the coupling between the models used in this study. All couplings take place through the Space Weather Modeling Framework. Red arrows denote couplings that are used in this study, and gray arrows denote couplings required to achieve two-way coupling between the ring current and the rest of the magnetosphere/ionosphere system (not used in this study). The large blue arrow represents outflow from the ionosphere through the magnetosphere and to the ring current.

electron temperature over the polar cap at $1600 \mathrm{~km}$ altitude. This particular example uses a statistical precipitation pattern based on the work by Fuller-Rowell and Evans [1987]. Cusp precipitation, and hence the associated heat flux, is not included in this plot. As expected, higher electron temperatures are seen both in the auroral region and on the sunlit dayside. Because the electron temperature is instrumental in determining the scale height of the ions, this process is a major and needed improvement to the model. Unfortunately, the topside electron energy flux is poorly understood, so a more complicated relationship is not instituted at this time.

[12] The BATS-R-US code [Powell et al., 1999; De Zeeuw et al., 2000] is the global MHD model used in this study. This model has a strong history of terrestrial magnetosphere investigations [e.g., Gombosi et al., 1998; Ridley et al., 2002; Tóth et al., 2007; Zhang et al., 2007] and validation [Yu and Ridley, 2008; Wang et al., 2008; Welling and Ridley, 2010a]. It uses an adaptive Cartesian grid in geocentric solar magnetospheric (GSM) coordinates. The inner boundary is a sphere of radius $2.5 R_{E}$; the outer boundary is $32 R_{E}$ in the upstream direction, $224 R_{E}$ downstream, and $128 R_{E}$ in each other direction. Observed upstream solar wind conditions are imposed as the upstream boundary conditions. The grid resolution used in this study yields approximately 1.9 million grid cells. It is finest near the Earth $\left(1 / 8 R_{E}\right)$; geosynchronous orbit lies in a $1 / 4 R_{E}$ resolution region that ranges from +8 to -20 in the $x$ direction and \pm 8 in the $y$ and $z$ directions.

[13] Two versions of BATS-R-US are used in this study: the standard single species/single fluid version and a newer single fluid/multispecies version. The multispecies version [Ma et al., 2002] divides the mass density into different species by including a separate continuity equation for each. This allows the different populations to be traced through the simulation domain. However, there remains only a single momentum and energy equation, so the solution is still, essentially, single fluid. The multispecies simulations used in this study define three species: protons $\left(\mathrm{H}^{+}\right)$, Helium ions $\left(\mathrm{He}^{+}\right)$, and oxygen ions $\left(\mathrm{O}^{+}\right)$. The densities and total plasma velocity at the inner boundary is set by the PWOM. The single species version follows only $\mathrm{H}^{+}$, so no composition information is tracked.

[14] The inner boundary is an important source for plasma in BATS-R-US. Without the PWOM, the inner boundary is a sphere of constant mass, so outflow is the result of pressure gradients pulling this mass into the domain. Welling and Ridley [2010b] used multispecies BATS-R-US to track this default outflow and found it to be the dominant source of plasma in the inner magnetosphere and central plasma sheet during periods of southward interplanetary magnetic 


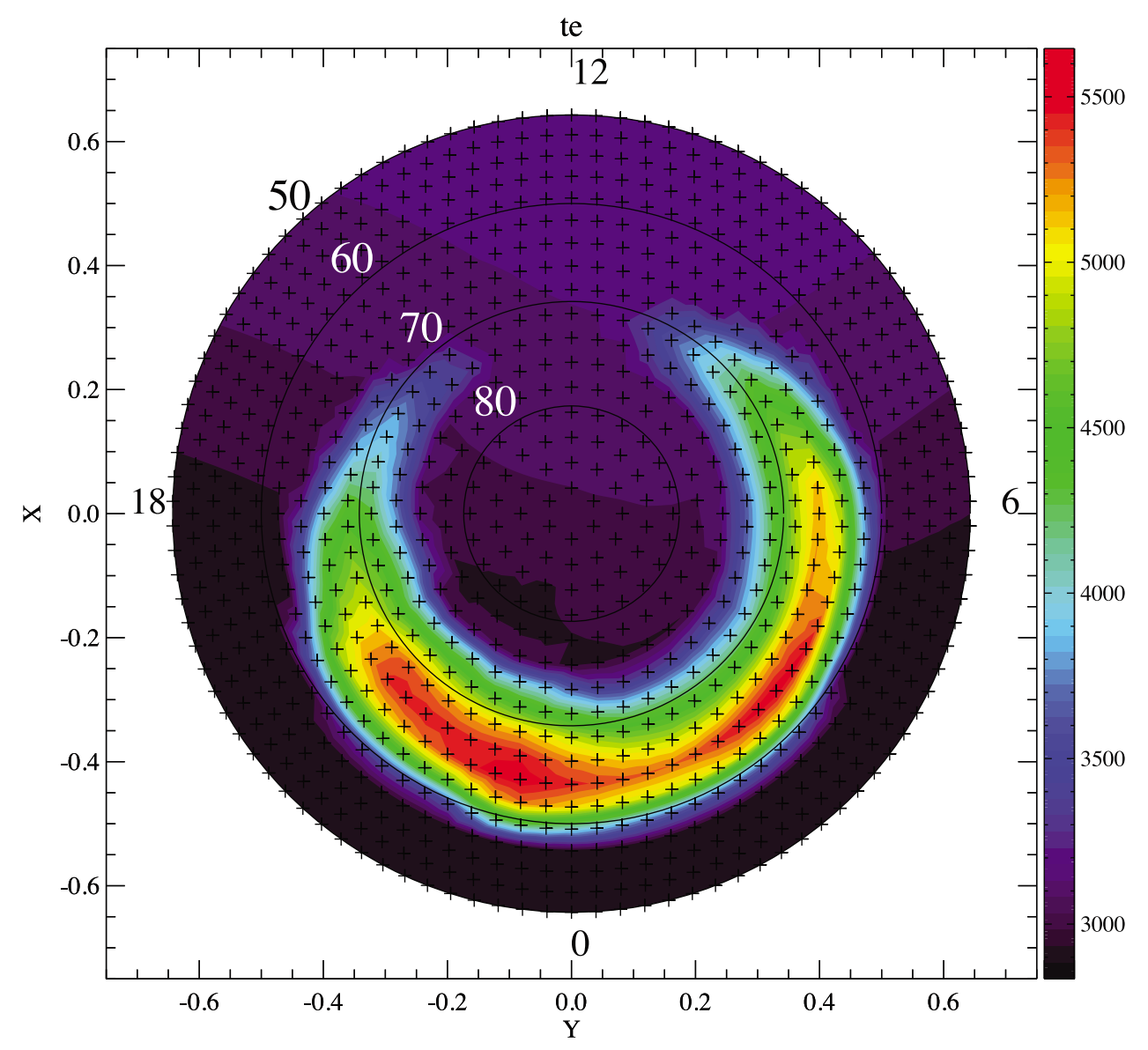

Figure 2. A typical electron temperature calculation in PWOM using the new method for specifying the topside electron heat flux. The color contour is the temperature in Kelvin, and the plus signs show the locations of the field line foot points in invariant latitude of field lines in the PWOM calculation.

field (IMF). During periods of northward IMF, solar wind plasma entering through the flanks was the dominant source. When adding PWOM to the modeling suite, Glocer et al. [2009a, 2009b] arrived at similar conclusions, noting the strong $\mathrm{O}^{+}$concentrations in the same regions.

[15] Interestingly enough, changing the outflow in BATSR-US does not seem to have a great effect on the global magnetospheric configuration. Without PWOM, the inner boundary density is typically set at $28 \mathrm{~cm}^{-3}$. Welling and Ridley [2010b] noted that this is much higher than observations reported by Huddleston et al. [2005]; both Zhang et al. [2007] and Welling and Ridley [2010b] found that changing this value has only a marginal effect on the results. Glocer et al. [2009a] came to the same conclusion. It is only when an inner magnetosphere code is two way coupled to this system, receiving mass from BATS-R-US and returning plasma pressure to increase the values calculated by MHD, that the increased mass outflow provided by PWOM can significantly change magnetospheric configuration [Glocer et al., 2009a].

[16] BATS-R-US sends field aligned currents to RIM [Ridley and Liemohn, 2002; Ridley et al., 2004] in order to handle the ionospheric electrodynamics. RIM uses these to calculate particle precipitation and conductance patterns. The conductance and field-aligned currents are used to calculate the electric potential, which is in turn mapped back to the inner boundary of BATS-R-US. The electric potential is also used by PWOM to advect magnetic field lines.

[17] The output of these models is coupled one way in order to drive the RAM-SCB model. This code couples two separate models: a kinetic model and a 3-D force balance magnetic field model. Coupling these two produces self consistency between the particles drifting in the ring current and the magnetic field through which they are drifting.

[18] The kinetic model is the Ring current Atmosphere interaction Model, or RAM [Jordanova et al., 1996, 1997], which solves the kinetic equation to yield the bounceaveraged distribution function as a function of azimuth, radial distance, energy and pitch angle for three ion species $\left(\mathrm{H}^{+}, \mathrm{He}^{+}\right.$, and $\left.\mathrm{O}^{+}\right)$. The domain is a circle in the solar magnetic (SM) equatorial plane with a radial span of 2 to $6.5 R_{E}$. It has an energy range of approximately $100 \mathrm{eV}$ to $500 \mathrm{KeV}$. It includes charge exchange losses, Coulomb collision losses, and atmospheric loss at low altitudes. The distribution function at the outer boundary is set by observations or separate model results; the inner boundary holds the distribution function constant at zero. The model was recently updated to use nondipolar field geometries [Jordanova et al., 2006, 2010]. 


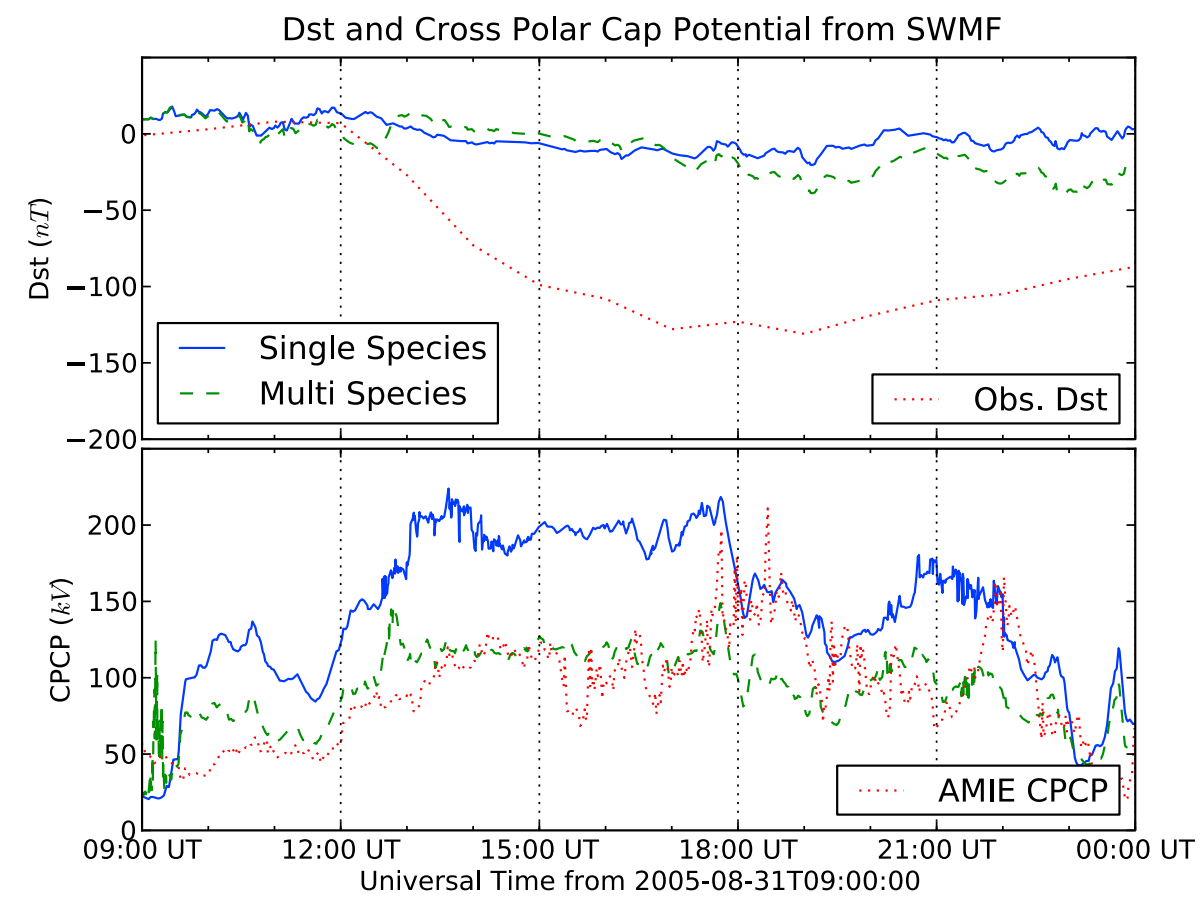

Figure 3. A comparison between the single species MHD simulation without PWOM (blue solid lines) and the multispecies simulation with PWOM (green dashed lines) in terms of (top) Dst index and (bottom) cross polar cap potential. For reference, observed Dst (Figure 3, top, red dotted line) and AMIE CPCP (Figure 3, bottom, red dotted line) are also included.

[19] The other half of RAM-SCB is the 3-D force balance magnetic field model [Zaharia et al., 2004; Zaharia, 2008]. This model balances the $\mathbf{J} \times \mathbf{B}$ force with the divergence of the general pressure tensor to calculate the magnetic field configuration within its domain. The domain ranges from near the Earth's surface, where the field is assumed dipolar, to the shell created by field lines passing through the SM equatorial plane at a radial distance of $6.5 R_{E}$. Anisotropic pressure both at the outer boundary and inside the code's domain is required. By relying on anisotropic pressure calculated by RAM, the force balance model creates a more stretched, more realistic field than isotropic MHD models that do not capture the ring current pressure build up and are typically very dipolar within $6.6 R_{E}$.

[20] Initial coupling of these two codes is detailed in the work by Zaharia et al. [2005], Jordanova et al. [2006], and Zaharia et al. [2006]; details about the full coupling can be found in the work by Zaharia et al. [2010]. RAM provides anisotropic pressure to the 3-D equilibrium code, which in return calculates the field aligned integrals required by RAM to calculate particle drift paths. The addition of self consistency creates significant differences in the ring current drift paths [Jordanova et al., 2006] and a depression in the nightside magnetic field [Zaharia et al., 2006].

[21] In this work, all of the inputs required by RAM-SCB are provided by the other coupled codes. Convection electric field created by RIM is mapped along field lines calculated by RAM-SCB to obtain these values in the SM equatorial plane. The plasma density and pressure at the outer boundary of RAM-SCB (a circle of radius $6.5 R_{E}$ lying in the SM equatorial plane) are extracted from BATS-R-US and used to produce the particle flux assuming a Maxwellian, isotropic particle distribution. When using multispecies MHD and the PWOM, composition is obtained directly from the model results. Otherwise, the empirical formula given by Young et al. [1982] (dependent on F10.7 flux and Kp index) is used to divide the MHD mass density into number density for each of the three species required. Both methods conserve MHD mass density when calculating number density. Temperature is obtained from the MHD pressure by using the number density obtained after determining the composition. Magnetic field boundary conditions for the equilibrium code are taken from BATS-R-US; the process for computing the field shell is outlined by Zaharia et al. [2010].

[22] To simplify this work, the same magnetic field boundary conditions are used for all the RAM-SCB simulations and are taken from the single species, no PWOM MHD simulation. This allows for a cleaner interpretation of the results. This simplification likely has a minimal impact, however, given that changing the outflow without a twoway coupled inner magnetosphere code has minimal impact on the MHD field configuration [Zhang et al., 2007; Glocer et al., 2009a; Welling and Ridley, 2010b]. Two papers complementary to this work fully explore the issue of different magnetic field configurations on the ring current [Jordanova et al., 2010] and different outer boundary conditions on RAM-SCB results [Zaharia et al., 2010].

[23] Using this setup, the 31 August 2005 coronal mass ejection (CME) event, a moderate storm whose minimum hourly measured Dst was -131 (Figure 3, top), was modeled using two different approaches. The first simulation was performed using a single species, no PWOM approach. The second used the multispecies approach with PWOM in order to include realistic oxygen delivery to the ring current. The 


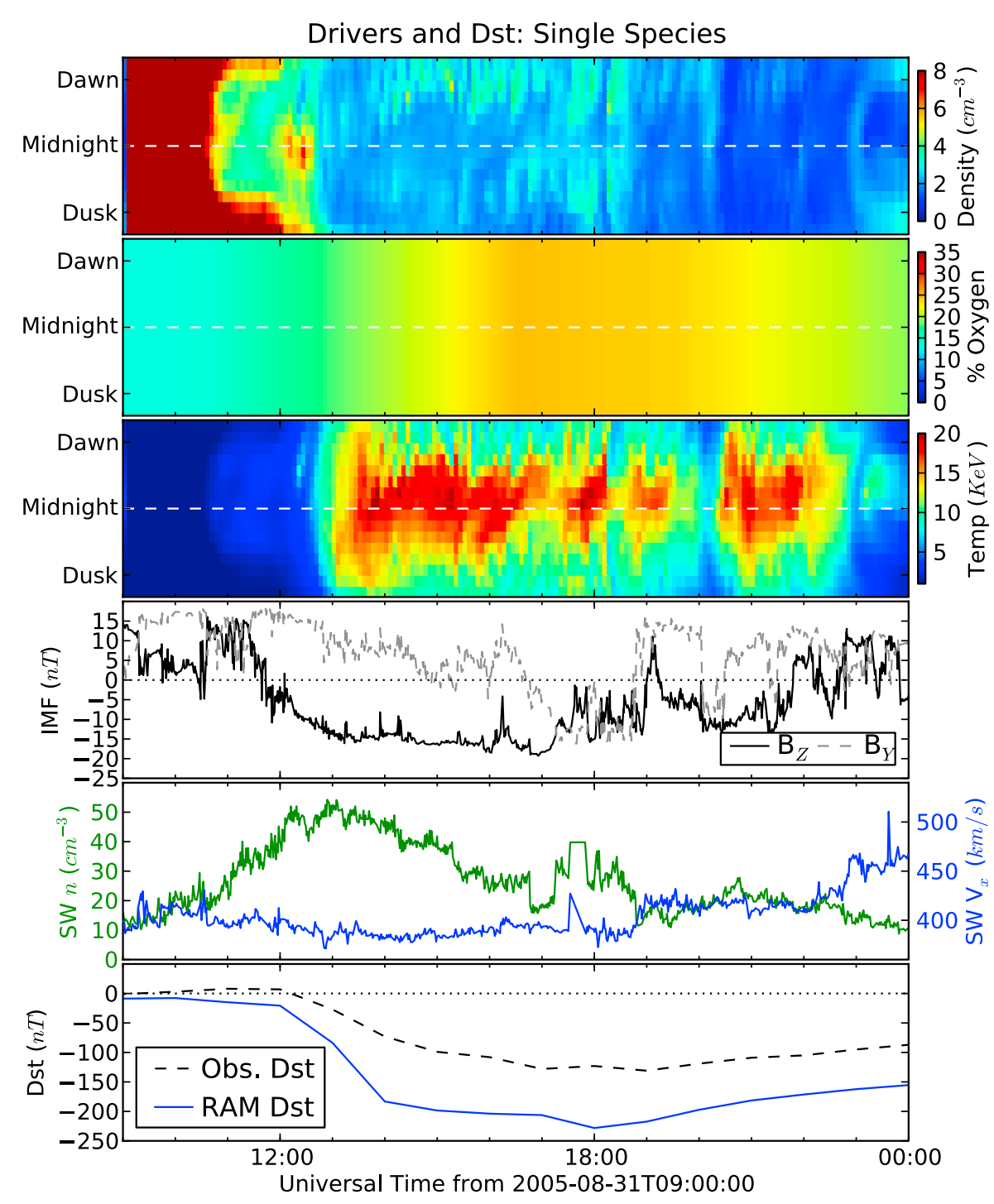

Figure 4. Summary of the single species, no PWOM ring current drivers and resulting Dst. The top three frames are local time-universal time maps of total number density, percent oxygen by number, and temperature, respectively. These values are the plasma sheet conditions that feed RAM-SCB. The fourth and fifth frames show IMF and solar wind drivers (as labeled). The bottom frame compares the resulting RAM-SCB calculated Dst to the observed Dst.

two sets of results were compared against each other to identify the main differences between ring current drivers with and without the dynamic, causal ionospheric outflow. The effects of these drivers on the ring current were examined through the RAM-SCB results. These simulations were repeated with artificial changes to the drivers in order to isolate the key driver-effect relationships. While these artificial changes are a powerful way to further probe the relationship between ionospheric outflow and ring current development, it breaks the self-consistency of the calculated drivers and should be interpreted with this in mind.

\section{Results}

[24] Figure 3 (top) shows the Dst resulting from the single species global simulation without driven outflow (blue solid line) and the multispecies global simulation with driven outflow (green dashed line) along with the measured Dst (red dotted line) for comparison. Dst in BATS-R-US is calculated through a whole domain Biot-Savart integral at the center of the Earth; the performance without a two-way coupled inner magnetosphere model is typically very poor [Zhang et al., 2007]. This is the case for both of these simulations. Overall, the Dst values for both are similar throughout the main phase of the storm. Though the two curves begin to diverge after IMF $B_{Z}$ turns northward, this plot supports the assumption that the magnetospheric configuration, especially in the inner magnetosphere, for both simulations is very similar. The magnetic elevation angle at $\mathrm{X}=-6.6 R_{E}$ at $1700 \mathrm{UT}$ is approximately $54^{\circ}$ for both cases, demonstrating that this is especially true near the outer boundary of RAM-SCB.

[25] The results of the single species BATS-R-US simulation at a circle of radius $6.5 R_{E}$ are summarized in Figure 4. 
These are the plasma sheet values used to set the outer boundary conditions of RAM-SCB. The top panel is a local time-universal time map of the total plasma number density. Each vertical slice yields the number density at a single epoch but a range of local times, from local dusk (bottom of frame) to local midnight (middle of frame) to local dawn (top of frame), all at a constant radial distance of $6.5 R_{E}$. Corresponding maps of composition as percent oxygen ions by number density and total plasma temperature are shown in the second and third frames from the top. The next two panels show the solar wind drivers: IMF (fourth from top, $B_{Z}$ in solid black and $B_{Y}$ in dashed grey) and solar wind number density and earthward velocity (second from bottom).

[26] The prevailing, macroscale behavior of the plasma sheet along the outer boundary of RAM-SCB follows the patterns described by Welling and Ridley [2010b]. This work found that in BATS-R-US, southward IMF drives magnetic convection that brings plasma from the inner boundary to the plasma sheet and heats it adiabatically as it is brought earthward. During northward IMF conditions, the ionospheric plasma (rather, the plasma originating from the inner boundary) becomes cut off from the inner magnetosphere. Solar wind plasma becomes the dominant source as it enters through the flanks. This plasma experiences little adiabatic heating leading to a colder, denser plasma sheet. Depending on the strength and direction of IMF $B_{Z}$ as well as the solar wind ram pressure, these two processes act in balance of each other to populate the plasma sheet. These general patterns are found in Figure 4. Around 1200 UT, the IMF turns and remains southward. Following this, around $1230 \mathrm{UT}$, the plasma sheet density drops while the temperature increases. At 1900 UT, a short-lived northward turning manages to cool the plasma at 1930 UT. The IMF turns southward again, weakly at 1930 then strongly at 2000 UT, then back northward at 2140. The plasma sheet temperature responds to each of these turnings, again with a delay of 20-40 minutes. The delay is due to required travel time from the code's dayside boundary to the magnetopause and as well as the time it takes for the magnetospheric response to propagate to and around the $6.5 R_{E}$ circle.

[27] There are regions where this pattern does not hold, particularly at 1200,2000 , and 2250 UT. Each of these epochs is marked by an increase in number density; the latter two have a corresponding drop in plasma sheet temperature. These events are caused by the release of plasmoids from the MHD magnetotail with corresponding dipolarizations of the closed field lines. Such events drive cold, dense plasma injections at $6.6 R_{E}$. The reason the plasma remains cold is very likely due to the lack of accurate reconnection physics in ideal MHD, but further investigation of this and a contrast of these events to observed magnetospheric substorms is beyond the scope of this paper.

[28] The one stand out feature of Figure 4 is the composition. Because it is set by the empirical formulation derived by Young et al. [1982], it varies slowly temporally and not at all spatially. oxygen makes up at least $\sim 13 \%$ of the total number density at all times and at most $\sim 25 \%$, ramping up then decreasing slowly throughout the main phase of the storm. These features are far less dynamic than those of the number density and temperature. The empirical composition does not show the responsiveness to the solar wind drivers displayed by the MHD-derived values (density and temperature).
[29] The bottom frame of Figure 4 uses the Dst index to summarize the results of using these outer boundary conditions to drive RAM-SCB. The measured hourly index is shown as a black dashed line, the Dst from RAM-SCB, computed through the Dessler-Parker-Sckopke relation [Dessler and Parker, 1959; Sckopke, 1966], including currents induced in the diamagnetic Earth, is shown as a solid blue line. RAM-SCB over estimates $D s t$ strongly. This is most likely due to the dense plasma that is provided by BATS-R-US.

[30] Figure 5 is the same as Figure 4, but for the global simulation using multispecies MHD and driven outflow using PWOM. The overall trends found in the single species case are seen here, such as the density drop and temperature increase as IMF turns southward at storm onset and the cooling/warming of the plasma sheet with subsequent turnings. Further similarities are seen in the plasmoid/ dipolarization events, observed in both simulations. Each corresponds to a dropout in oxygen, implying a solar wind source [Welling and Ridley, 2010b] that would explain the associated temperature drop. The final event (2240 UT) is far more pronounced than its single fluid counterpart. Again, these issues are left to future studies that focus on substorm dynamics and their MHD analogs.

[31] The percent $\mathrm{O}^{+}$rises and falls through the main phase of the storm, roughly the same as the empirically driven case. However, the variability of composition is much stronger than that of the empirically set simulation. At many times the oxygen contribution is less than the empirically set case, but at other times it is much more. The temporal differences are compounded by spatial ones as the composition is now local time-dependent.

[32] There appears to be an IMF $B_{y}$ effect, as the periods of strong positive (negative) $B_{y}$ correlate with periods of stronger oxygen contributions on the duskside (dawnside). For example, around $1520 \mathrm{UT}, B_{y}$ becomes moderately positive. Shortly after, beginning at 1600 UT, a strong injection of $\mathrm{O}^{+}$arrives, but is concentrated duskward of local midnight. As $B_{y}$ becomes negative (1700 to $1800 \mathrm{UT}$ ), the strongest contribution of oxygen moves toward the dawnside (1830 to $1900 \mathrm{UT}$ ). This again flips as $B_{y}$ turns positive at $1900 \mathrm{UT}$ with the local time peak of $\mathrm{O}^{+}$turning duskward approximately 20 minutes after. Again, there is a delay between the IMF at the upstream boundary of the code and when the plasma sheet responds. None of these new local and universal time complexities were obtained using the empirically driven composition.

[33] Other differences continue to set the two simulations apart. As the set density boundary conditions without PWOM are very high, $28 \mathrm{~cm}^{-3}$ [Welling and Ridley, 2010b], the total outflow is now lower with PWOM than without. Even so, the total mass density has increased because oxygen is now explicitly included. As such, the number density and temperature have decreased compared to the single fluid, no PWOM simulation. There are many small-scale differences in the overall patterns, likely due to the dynamic outflow strength and spatial dynamics produced by PWOM that are not obtained with a constant, smooth inner boundary. The end result of these differences is a RAM-SCB simulation that underpredicts the Dst index (Figure 5, bottom frame).

[34] Figure 6 illustrates the results of the RAM-SCB simulations more closely. For simplicity, the RAM-SCB 


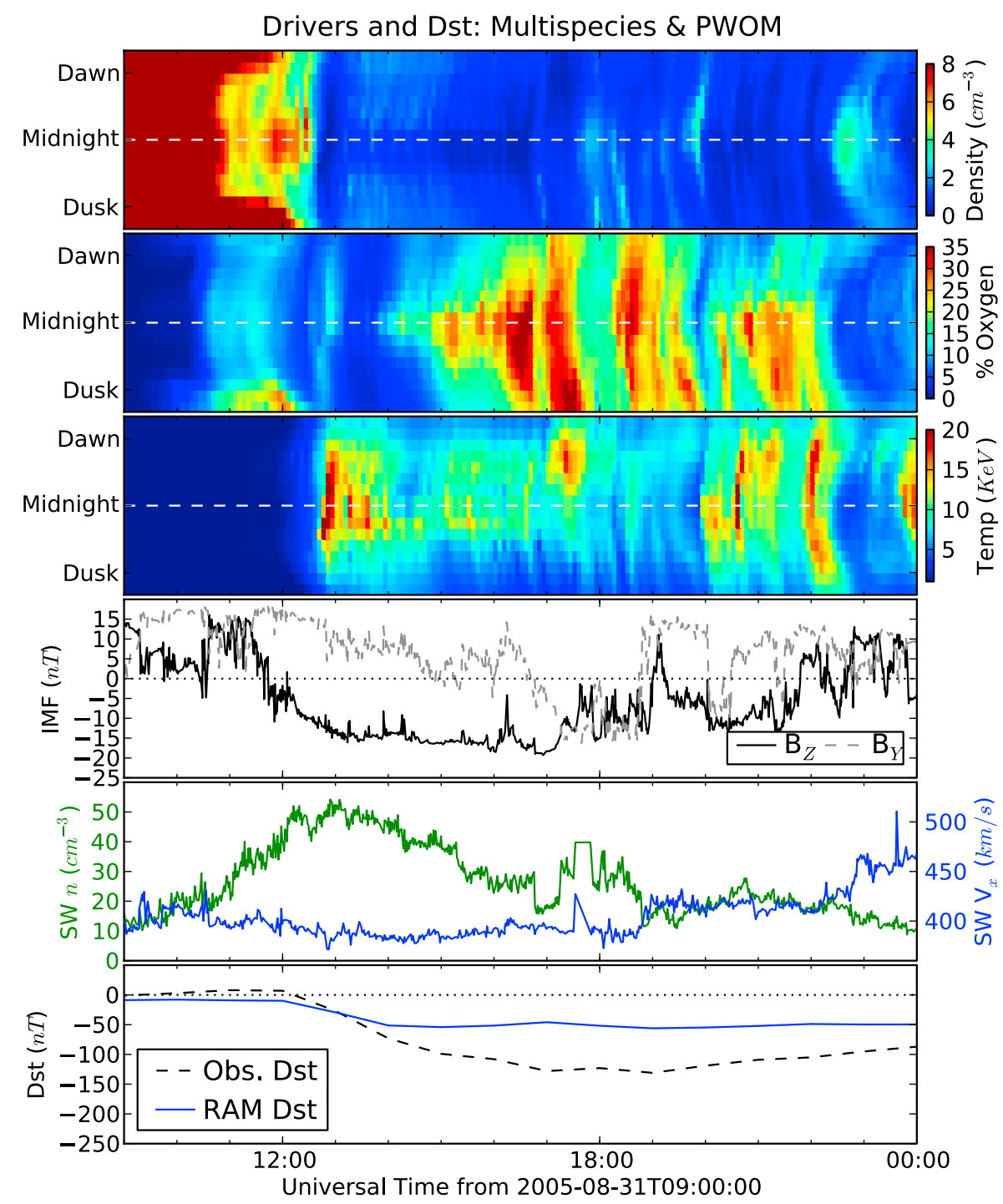

Figure 5. Same as Figure 4 but for the multispecies simulation with PWOM.

simulation driven by the single species MHD without PWOM is referred to as case 1; the simulation driven by multispecies MHD with PWOM is referred to as case 2. Cases 3 and 4, also shown in Figure 6, will be described later. Figure 6 (top) compares the calculated Dst from each of the first two simulations (case 1 in blue, case 2 in green, observed Dst in dashed black). The following grid of dial plots (Figure 6, bottom) shows the energy density as calculated by RAM-SCB as $\mathrm{H}^{+}$contribution and $\mathrm{O}^{+}$contribution pairs. Each pair column is taken from a different point in the storm: early storm (1400 UT), storm maximum (1700 UT), and early recovery (2300 UT). Each row is taken from a different simulation, with case 1 and case 2 on top and second from top, respectively.

[35] The two simulations show interesting differences throughout the main phase. At $1400 \mathrm{UT}$, it is already evident that the multispecies driven simulation (case 2) is much weaker. The pressure distributions cover a much narrower radial and local time range compared to case 1 . The $\mathrm{H}^{+}$and
$\mathrm{O}^{+}$maps appear to be scaled mirrors of each other (for the empirically set composition of the single species run, this is indeed the case.) At 1700 UT, subtle differences arise between the energy density maps of the two species in case 2 that are not present in case 1 results. These differences can be quantified by locating the peak of each map. In case 2, the $\mathrm{H}^{+}$energy peak is at a radial distance of $3 R_{E}$ and post noon $(1400 \mathrm{LT})$, while the $\mathrm{O}^{+}$peak is at $3.25 R_{E}$ and premidnight (2200 LT, an azimuthal difference of $\left.120^{\circ}\right)$. The peaks for case 1 simulation are both located at $3.25 R_{E}$ and a local time of 2200. Finally, at 2300 UT (Figure 6, right), the ring current has become much more symmetric for both simulations. At this point, the field configuration of the two global simulations begin to diverge, as seen in Figure 3. Both release a plasmoid down tail, resulting in a dipolarization of the field and a subsequent particle injection seen in the top frames of Figures 4 and 5, the latter being more pronounced. Returning to Figure 6, the injection is too small 


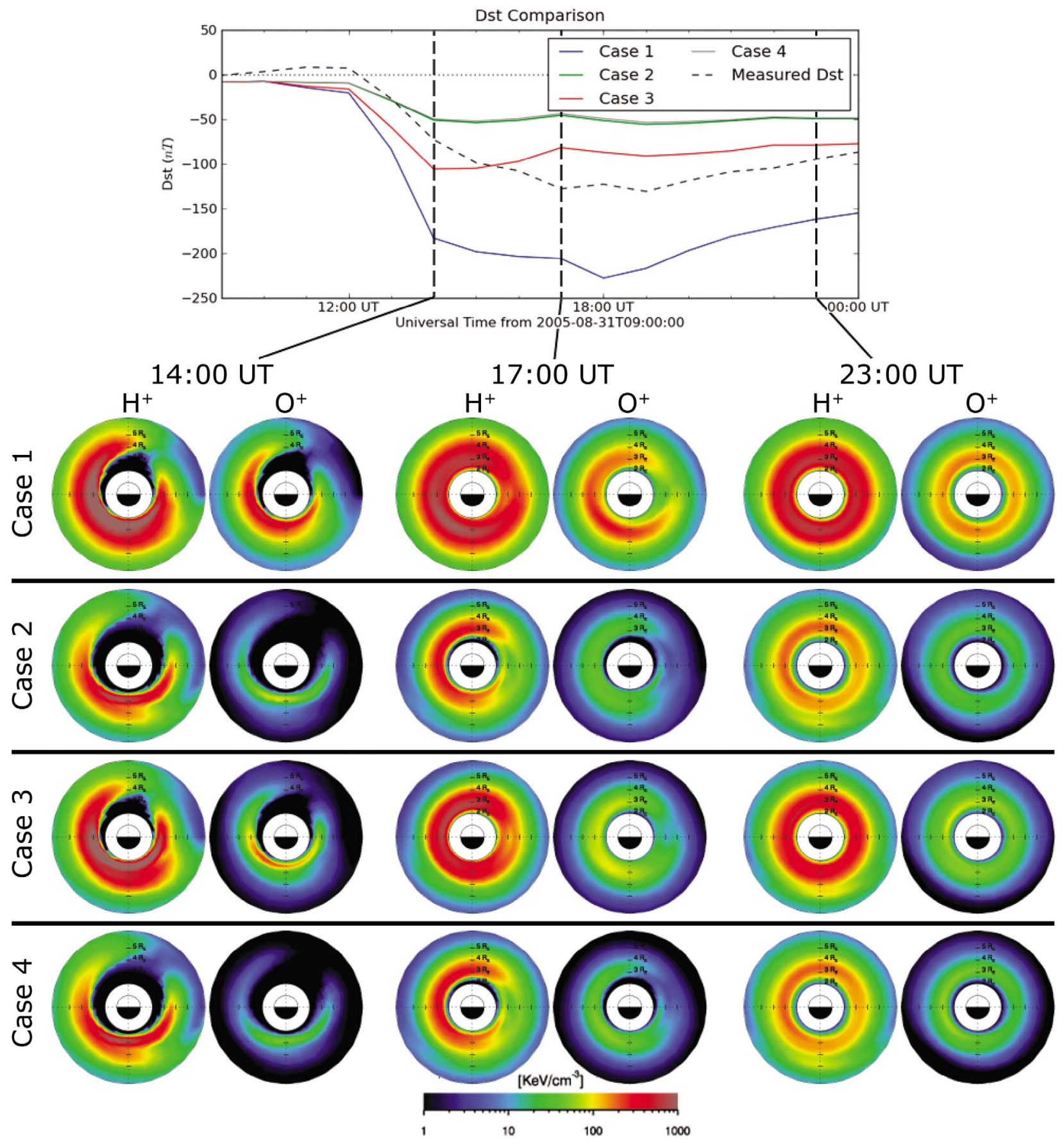

Figure 6. Comparison of different RAM-SCB simulations using (top) calculated Dst index and (bottom) global energy density distribution. The energy density plots come in $\mathrm{H}^{+}-\mathrm{O}^{+}$pairs to show the contribution of each species. The lines for case 2 and case 4 overlap and are nearly indistinguishable.

to notice in case 1 but manifests as a strong $\mathrm{H}^{+}$injection in case 2 results with no corresponding $\mathrm{O}^{+}$injection.

[36] The differences in the energy density maps do not correspond to great differences in the pitch angle distributions. Though some differences between the global distribution of anisotropies were found, these were minor and uninteresting. This is mostly due to the assumption of isotropy that is made when calculating fluxes from the MHD code at the outer boundary of RAM-SCB.

\subsection{Electric Field}

[37] It is clear that many of the ring current differences between case 1 and case 2 can be tied to the differences in plasma number density and temperature (thus, flux) provided to the outer boundary of RAM-SCB, but there are other effects that must be considered as well, such as the local time dynamics of $\mathrm{O}^{+}$delivery. The question of the relevance and importance of each effect rises. It is possible 
to artificially change the RAM-SCB drivers to separate and assess each. While doing so breaks the self consistency inherent in the creation of the drivers, it is still a useful exercise for testing whether such effects are relevant and their relative importance.

[38] One such effect is the reduced electric field resulting from the inclusion of PWOM in the global simulation. Figure 3 (bottom) shows the cross polar cap potential (CPCP) for both simulations. When PWOM is added in case 2 , the CPCP is decreased by as much as $100 \mathrm{kV}$. This effect has been observed by both Glocer et al. [2009a] and Winglee et al. [2002]. To examine how reducing the convection electric field impacts the ring current, the RAM-SCB portion of case 2 was repeated, but the electric field from case 1 was imposed instead of the weaker one produced in case 2.

[39] The results of this new simulation, case 3, are shown in Figure 6, both in the top Dst plot and the third row of energy density dial plots. The Dst is shown as a red line; the combination of the enhanced convection electric field and the PWOM outflow yields the best comparison to the measured Dst. This may be misleading, however, because contributions to the Dst outside of the RAM-SCB domain are not included in the calculation. Such contributions can be significant [Turner and Baker, 2000; Ohtani et al., 2001; Ganushkina et al., 2004], pushing the Dst from case 2 toward more realistic values. The ring current, for both species shown, has been intensified significantly from case 2 but has not reached the overdriven levels of case 1 . The shape is similar to that of case 2 .

[40] Figure 7 explores the differences when using the stronger electric field more deeply. Each frame shows the omnidirectional flux for $\mathrm{H}^{+}$and $\mathrm{O}^{+}$pairs at local noon, $\mathrm{R}=$ $3 R_{E}$ (Figure 7, top) and $\mathrm{R}=5 R_{E}$ (Figure 7, bottom). Local noon is chosen to clearly show fluxes of plasmas that have evolved through the inner magnetosphere. Case 2 is shown in Figure 7 (left), and case 3 is shown in Figure 7 (middle).

[41] During the first half of case 2, the storm time plasma has not reached the dayside yet. The initial condition material is still advecting through the code, so the two species look very independent of each other. Around 1400 UT, storm time plasma arrives. Both cooler, $\mathbf{E} \times \mathbf{B}$ as well as warmer, gradient curvature drifting plasma is observed with a clear stagnation point located between the two. The warmer plasma arrives earlier, especially at $\mathrm{R}=3 R_{E}$. At this point, it becomes clear that the two species are strongly tied to each other due to the multispecies, single fluid approach: because each species has the same temperature, only the magnitude is different, not the overall pattern.

[42] Increasing the convection electric field, as is done in case 3 , brings the cooler plasma to the dayside faster and increases the width of the captured warm band. The reasons are intuitive: the stronger electric field is able pull warmer plasma deeper into the inner magnetosphere while accelerating colder plasma. In doing so, the clear stagnation point seen in case 2 is now blurred. The net effect is a stronger, more energetic ring current.

\subsection{Species Temperatures}

[43] Figure 7 demonstrates an important issue when using a multispecies, single fluid approach. The RAM-SCB results for each species can differentiate themselves in terms of magnitude because they have different densities at the outer boundary. However, because they have the same temperature, the energy distributions are still tied to one another. This results in limited differences between the two species.

[44] To examine how removing this limitation may affect the results here, case 2 was repeated, but the $\mathrm{O}^{+}$temperature is reduced by $50 \%$. Fluxes are recomputed, and RAM-SCB is rerun, producing the case 4 results shown in Figure 6. The Dst computed from this simulation is shown as a gray line, but it is nearly impossible to distinguish from the Dst of case 2 . The energy density dial plots for this simulation, seen in Figure 6 (bottom), show some minor differences compared to case 2 , but these are minimal. Omnidirectional flux for case 4 is shown in Figure 7 (right). As expected, there are no differences in the $\mathrm{H}^{+}$fluxes from case 2 , but clear changes in the $\mathrm{O}^{+}$fluxes. At $\mathrm{R}=3 R_{E}$, there is an increase in the captured cold flux. At $\mathrm{R}=5 R_{E}$, the gradient curvature drifting population is colder and weaker with a corresponding slight increase in the $\mathbf{E} \times \mathbf{B}$ population. Clearly, the differences in particle distributions do not translate into strong changes in the global ring current development. From these results, it does not appear that separating the species temperatures by lowering the $\mathrm{O}^{+}$temperature has an important effect. The effects may become more substantial in storms that have higher $\mathrm{O}^{+}$concentrations.

\subsection{Local Time of Injections}

[45] The final impact of first-principles-based outflow and delivery to the ring current is that of the differences in local time of the $\mathrm{O}^{+}$concentrations. While case 1 delivers $\mathrm{O}^{+}$as a uniform percentage of the total number density, case 2 delivers it in a spatially dynamic manner. This results in periods of $\mathrm{O}^{+}$concentrations at $6.5 R_{E}$ that are sometimes centered at midnight and sometimes concentrated toward the dawnside or duskside. Such asymmetries can inject the plasma on very different drift paths and can potentially affect the development of the storm time ring current [Jordanova, 2006; Lavraud et al., 2008].

[46] To investigate this impact, focus returns to the case 1 simulation where the $\mathrm{O}^{+}$concentration is uniform and simple to manipulate. The concentration is changed to only allow $\mathrm{O}^{+}$to be injected into different local time windows by reducing the total number density outside of the window to a very small value. Three windows were used: a dusk window (1800-2100 LT), a midnight window (2200-0200), and a dawn window (0300-0600). These edited boundary conditions are used to perform three new RAM-SCB simulations.

[47] Figure 8 compares the results from these three simulations. The Dst for each, shown in Figure 8 (top), are similar, but the dusk case is noticeably weaker than the other two. Below the Dst plot are $\mathrm{O}^{+}$energy density plots at 1700 UT for each case. The dusk case captures almost no oxygen, the midnight case captures significant $\mathrm{O}^{+}$over a broad spatial range, and the dawn case captures even more $\mathrm{O}^{+}$over a narrow radial range.

[48] The explanation for the results shown in Figure 8 is intuitive. For the dusk case, the oxygen ions are injected on open drift paths and immediately gradient curvature drift out of the simulation domain. In the other two cases, more plasma is captured on closed drift paths, retaining more energy in the ring current. The dawn case injects the $\mathrm{O}^{+}$into 


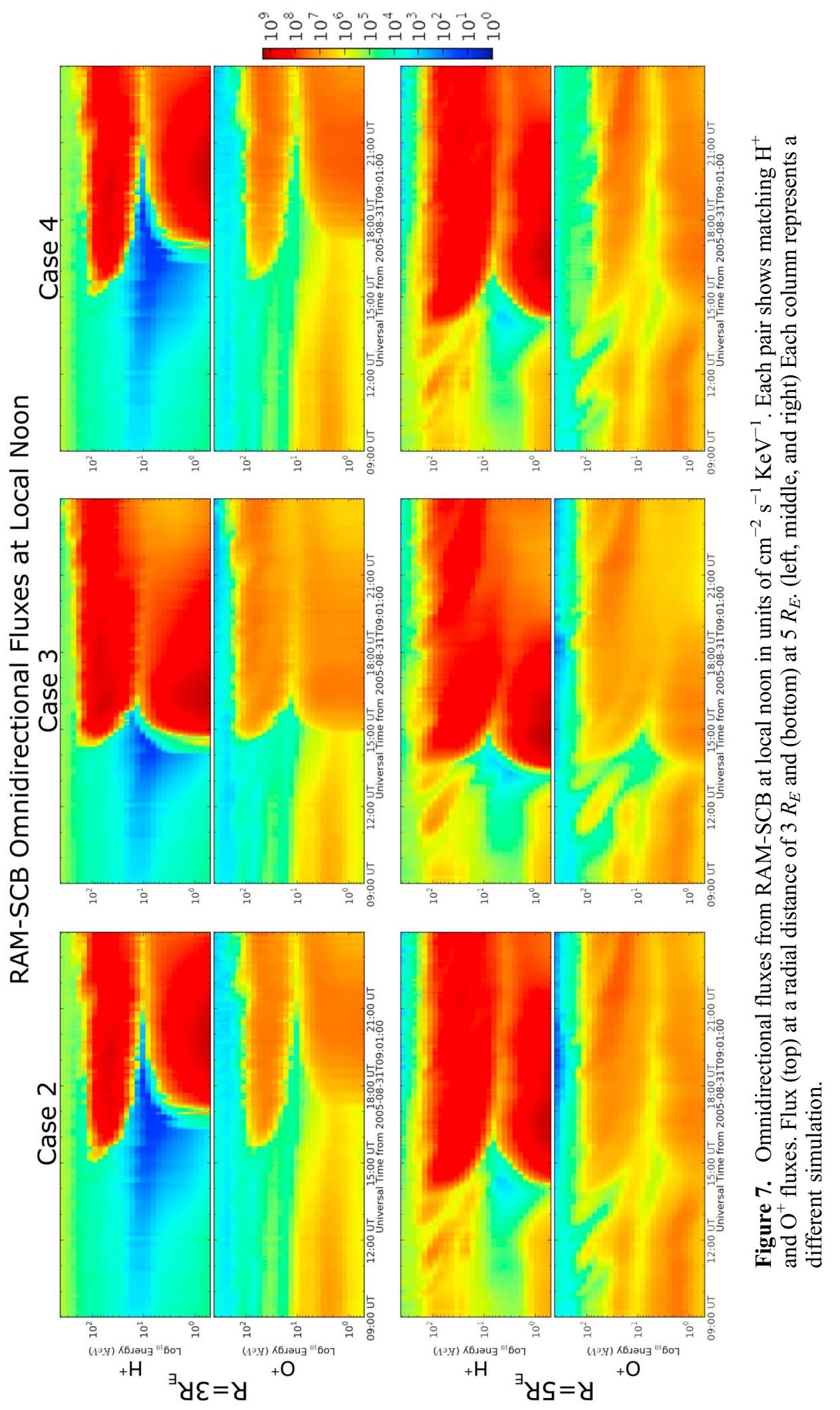




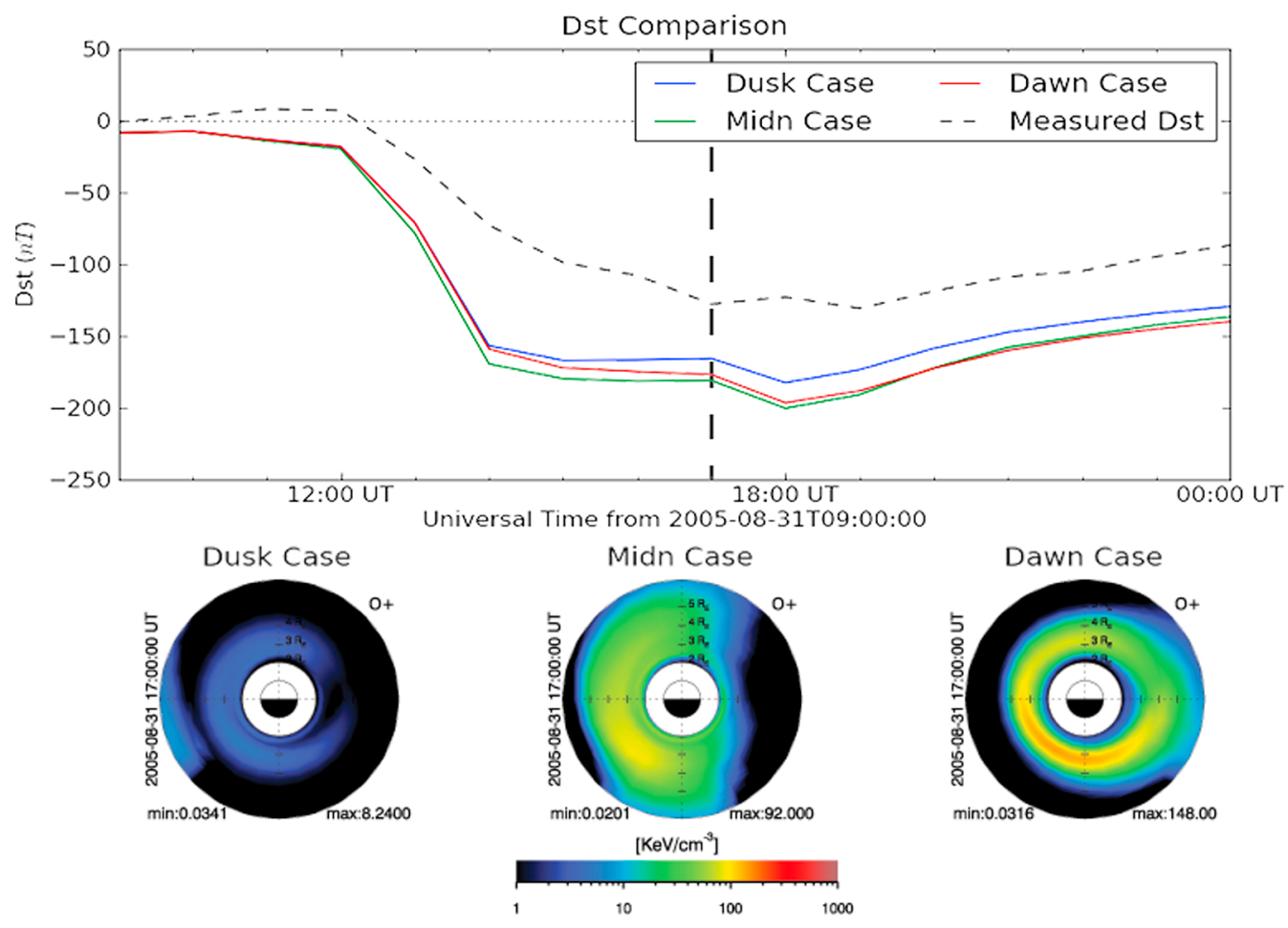

Figure 8. Comparison between three RAM-SCB simulations: one where $\mathrm{O}^{+}$is relegated to a dusk local time window along the outer boundary (dusk case), one where it is limited to a midnight centered window (midnight case), and one where it is limited to a dawnside window (dawn case). (top) Comparison of the Dst calculated from each simulation. (bottom) The $\mathrm{O}^{+}$energy density at 1700 UT for each case is shown.

a narrower band than the midnight case. Based on these results, it is clear that the local time dynamics in the $\mathrm{O}^{+}$play a role in the development of the storm time ring current.

\section{Conclusions and Discussion}

[49] This study is the first to examine how first-principlesbased modeling of ionospheric outflow and its advection through the global magnetosphere affect the development of the main phase storm time ring current. Two global simulations were performed to produce a set of ring current drivers: one with single species MHD and ionospheric electrodynamics that uses an empirical formula to set the composition in the plasma sheet, another with single-fluid, multispecies MHD, ionospheric electrodynamics, and polar wind outflow. Each domain was coupled together to provide a level of self-consistency between the outflow and the delivery to the ring current through the magnetosphere. Several important differences in the ring current drivers were discovered when outflow was included versus when it was neglected.

[50] 1. The plasma sheet number density and temperature were reduced when dynamic outflow was included.

[51] 2. $\mathrm{O}^{+}$concentrations in the plasma sheet were far more dynamic spatially, temporally, and in magnitude than the case where composition was set by an empirical formula.

[52] 3. The cross polar cap potential drop is reduced significantly when first-principles outflow is included.

[53] The reasons for each of these are varied. The reduced number density may be due to an underproduction of out- flow in PWOM. Several mechanisms for the reduction of CPCP, hence the dayside reconnection rate, have been suggested. Winglee et al. [2002] suggested that the additional mass provided by $\mathrm{O}^{+}$outflow reduces the overall convection speed that can be obtained given the same amount of momentum flux provided by the solar wind. Glocer et al. [2009a] and Wiltberger et al. [2010] both went further, noting that the greatest mass increase is near the inner boundary of the MHD model, affecting the calculation of the current toward the inner boundary thus the electric potential in RIM. Finally, the local time variations appear to be tied to IMF $B_{y}$; positive $B_{y}$ pushing the stronger $\mathrm{O}^{+}$ concentrations toward the duskside, negative toward the dawnside. It is well known that a strong $B_{y}$ component can rotate the plasma sheet about the $x$ axis [Cowley, 1981; Brecht et al., 1981; Walker et al., 1998]; this may move the denser, oxygen-rich portions of the sheet in and out of the SM plane, leading to the asymmetries found here. $B_{y}$ also changes field aligned current and convection patterns [ $\mathrm{Lu}$ et al., 1994; Weimer, 1995] which may be changing the outflow pattern in PWOM. This, too, may lead to the asymmetric $\mathrm{O}^{+}$contribution to the plasma sheet. For each of these processes, further investigation is required.

[54] The impact of the first-principles obtained drivers on the ring current was found to be significant. The energy of the ring current was greatly reduced overall throughout the main phase of the storm. The new complexities in the composition transitioned to the spatial distribution of ring current energy and decoupled the contribution to the total energy by $\mathrm{O}^{+}$from $\mathrm{H}^{+}$. This was not so in the single- 
species, no dynamic outflow case. In both cases, particle energy spectra of $\mathrm{O}^{+}$and $\mathrm{H}^{+}$look very similar because both species have the same temperature at the outer boundary. This shortcoming cannot be overcome easily when using the single fluid, multispecies MHD approach.

[55] To further evaluate the impact of the second and third points listed above, additional simulations were performed that isolate each. It was found that reducing the cross polar cap potential has strong implications on the strength of the ring current by reducing the amount of warm plasma that is captured. It was also found that the local time of plasma injection had an impact, though to a lesser extent. The further duskward the plasma is injected at $6.5 R_{E}$, the more likely it is to gradient curvature drift out of the domain and not contribute to the ring current. These effects are not captured unless a whole-system, coupled, first-principlesbased approach is taken to produce input to the RAM-SCB code.

[56] These impacts are especially relevant for the 31 August 2005 event, the storm that is the focus of this study and of great interest to the community. As there is a nontrivial IMF $B_{y}$ component throughout the storm, the composition is asymmetric about local midnight. Much of the $\mathrm{O}^{+}$plasma is delivered toward the duskside, rendering it incapable of contributing to the overall ring current energy density. Additionally, it appears that the CPCP during this storm as derived by Assimilative Mapping of Ionospheric Electrodynamics (AMIE) [Ridley and Kihn, 2004] (Figure 3, bottom, dotted red line) is closer to that predicted by the multispecies and PWOM case (green dashed line) than the higher values created by the non-PWOM simulation (blue solid line). There are a few periods, however, where the AMIE CPCP reaches the level of the non-PWOM case (e.g., 1730 UT). These could cause injections that are missed using the case 2 CPCP that would strengthen the ring current and push the calculated Dst values toward the observed values. It is clear that both of these effects are important factors for correctly modeling this event.

[57] The methods used to isolate the impact of the substantial changes that result from including dynamic outflow do a good job of illustrating the individual effects. However, they break the self-consistency that existed in the creation of the original drivers. For example, using the increased convection electric field to drive RAM-SCB yet the original plasma boundary conditions neglects the effect that this increased potential would have on the rest of the system. A stronger $\mathbf{E} \times \mathbf{B}$ velocity delivered to PWOM would increase centrifugal acceleration [Cladis, 1986; Horwitz, 1987]; the associated increase in field aligned currents would increase the radial electron velocity in PWOM. Both lead to stronger outflow, increasing the number density at the outer boundary of RAM-SCB, possibly creating the same overprediction of the ring current strength that was seen in the single-species, no outflow case. As such, it is difficult to truly isolate each effect because of the feedback it will have on the global system. The lack of self-consistency must be kept in mind while interpreting these results.

[58] This applies to the ring current as well. The one way coupling used in this study neglects the many ways in which the ring current feeds back into the global system. It has been demonstrated that returning the pressure calculated by a ring current code to the global MHD model can stretch the global magnetic field to much more realistic values $[D e$ Zeeuw et al., 2004], even more so when dynamic outflow is included in the modeling suite [Glocer et al., 2009a, 2009b]. By pushing the nightside reconnection point downtail, increased adiabatic heating will occur, yielding warmer plasma being fed into RAM-SCB, changing the dynamics of the ring current yet again. Finally, region 2 field aligned currents add another dimension of complexities to the picture by changing the global electric field pattern in RIM and changing the outflow in PWOM. Future work to complete this coupling and investigate these new issues is vital for validating the results here and broadening our understanding of the system.

[59] A clear limitation both to this study and any using the single-fluid, multispecies approach is the single plasma temperature for all species. It is expected that, due to the geomagnetic spectrometry effect [Chappell et al., 1987], the heavier ions will arrive at the central plasma sheet nearer to the Earth than the lighter ions. This should lead to disparate levels of adiabatic heating in the MHD model [Welling and Ridley, 2010b], and thus different temperatures for each, but a single fluid approach is incapable of separating flows and temperatures. The ad hoc method used here of indiscriminately reducing $\mathrm{O}^{+}$temperature by $50 \%$ did not have a significant effect on the ring current, but leaves open questions concerning a realistic case where species temperatures are truly decoupled spatially and temporally. In the future, a multifluid approach will be required to thoroughly investigate the importance of this.

[60] Despite these shortcomings, it is clear that ionospheric outflow affects the development of the storm time ring current in several complicated ways. Outflow cannot be treated as a black box source of oxygen ions when modeling the inner magnetosphere. The whole system must be taken into account in order to capture all of the interworking dynamics.

[61] Acknowledgments. The authors thank Aaron Ridley for providing the AMIE data used in this study. Dst data was provided by the World Data Center for Geomagnetism, Kyoto, and the four Dst observatories (Kakioka, Honolulu, San Juan, and Hermanus). Solar wind measurements are from the ACE SWEPAM and MAG instruments and were provided by the NASA GSFC Coordinated Data Analysis Web. Work at Los Alamos was conducted under the auspices of the U.S. Department of Energy, with partial support from the IGPP program and NASA Living With A Star grants NNH09AL06I and NNH07AG24I and NSF Geospace Environment Modeling grants ATM0902941 and ATM0703210.

[62] Masaki Fujimoto thanks Ramon Lopez and another reviewer for their assistance in evaluating this paper.

\section{References}

Bekerat, H. A., R. W. Schunk, and L. Scherliess (2007), Estimation of the high-latitude topside electron heat flux using DMSP plasma density measurements, J. Atmos. Sol. Terr. Phys., 69, 1029, doi:10.1016/j. jastp.2007.03.015.

Brambles, O. J., W. Lotko, P. A. Damiano, B. Zhang, M. Wiltberger, and J. Lyon (2010), Effects of causally driven cusp $\mathrm{O}^{+}$outflow on the storm time magnetosphere-ionosphere system using a multifluid global simulation, J. Geophys. Res., 115, A00J04, doi:10.1029/2010JA015469.

Brecht, S., J. Lyon, J. Fedder, and K. Hain (1981), A simulation study of east-west IMF effects on the magnetosphere, Geophys. Res. Lett., 8, 397.

Chappell, C. R., T. E. Moore, and J. H. Waite Jr. (1987), The ionosphere as a fully adequate source of plasma for the Earth's magnetosphere, J. Geophys. Res., 92, 5896. 
Cladis, J. B. (1986), Parallel acceleration and transport of ions from polar ionosphere to plasma sheet, Geophys. Res. Lett., 13, 893, doi:10.1029/ GL013i009p00893.

Cowley, S. W. H. (1981), Magnetospheric asymmetries associated with the $y$-component of the IMF, Planet. Space Sci., 29, 79, doi:10.1016/00320633(81)90141-0.

Daglis, I. A., R. M. Thorne, W. Baumjohann, and S. Orsini (1999), The terrestrial ring current: Origin, formation, and decay, Rev. Geophys., 37, 407, doi:10.1029/1999RG900009.

Denton, M. H., M. F. Thomsen, H. Korth, S. Lynch, J. C. Zhang, and M. W. Liemohn (2005), Bulk plasma properties at geosynchronous orbit, J. Geophys. Res., 110, A07223, doi:10.1029/2004JA010861.

Dessler, A. J., and E. N. Parker (1959), Hydromagnetic theory of geomagnetic storms, J. Geophys. Res., 64, 2239.

De Zeeuw, D. L., T. I. Gombosi, C. P. T. Groth, K. G. Powell, and Q. F. Stout (2000), An adaptive MHD method for global space weather simulations, IEEE Trans. Plasma Sci., 28, 1956.

De Zeeuw, D. L., S. Sazykin, R. A. Wolf, T. I. Gombosi, A. J. Ridley, and G. Tóth (2004), Coupling of a global MHD code and an inner magnetospheric model: Initial results, J. Geophys. Res., 109, A12219, doi:10.1029/2003JA010366.

Fok, M.-C., R. A. Wolf, R. W. Spiro, and T. E. Moore (2001), Comprehensive computational model of the Earth's ring current, J. Geophys. Res. 106, 8417, doi:10.1029/2000JA000235.

Fok, M.-C., T. E. Moore, P. C. Brandt, D. C. Delcourt, S. P. Slinker, and J. A. Fedder (2006), Impulsive enhancements of oxygen ions during substorms, J. Geophys. Res., 111, A10222, doi:10.1029/2006JA011839.

Fuller-Rowell, T. J., and D. S. Evans (1987), Height-integrated Pedersen and Hall conductivity patterns inferred from TIROS-NOAA satellite data, J. Geophys. Res., 92, 7606.

Gagne, J. (2005), Implementation of ionospheric outflow in the LFM global MHD magnetospheric simulation, M.S. thesis, Darthmouth College, Hanover, N. H.

Ganushkina, N. Y., T. I. Pulkkinen, M. V. Kubyshkina, H. J. Singer, and C. T. Russell (2004), Long-term evolution of magnetospheric current systems during storms, Ann. Geophys., 22, 1317.

Glocer, A., T. I. Gombosi, G. Toth, K. C. Hansen, A. J. Ridley, and A. Nagy (2007), Polar wind outflow model: Saturn results, J. Geophys. Res., 112, A01304, doi:10.1029/2006JA011755.

Glocer, A., G. Tóth, T. Gombosi, and D. Welling (2009a), Modeling ionospheric outflows and their impact on the magnetosphere, initial results, J. Geophys. Res., 114, A05216, doi:10.1029/2009JA014053.

Glocer, A., G. Tóth, Y. Ma, T. Gombosi, J.-C. Zhang, and L. M. Kistler (2009b), Multifluid Block-Adaptive-Tree Solar wind Roe-type Upwind Scheme: Magnetospheric composition and dynamics during geomagnetic storms-Initial results, J. Geophys. Res., 114, A12203, doi:10.1029/ 2009JA014418.

Gombosi, T. I., and A. Nagy (1989), Time-dependent modeling of field aligned current-generated ion transients in the polar wind, J. Geophys Res., 94, 359.

Gombosi, T. I., D. L. De Zeeuw, C. P. T. Groth, K. G. Powell, and P. Song (1998), The length of the magnetotail for northward IMF: Results of 3D MHD simulations, in Physics of Space Plasmas, vol. 15, edited by T. Chang and J. R. Jasperse, p. 121, MIT Press, Cambridge, Mass.

Horwitz, J. L. (1987), Core plasma in the magnetosphere, Rev. Geophys., 25,579

Huddleston, M. M., C. R. Chappell, D. C. Delcourt, T. E. Moore, B. L. Giles, and M. O. Chandler (2005), An examination of the process and magnitude of ionospheric plasma supply to the magnetosphere, J. Geophys. Res., 110, A12202, doi:10.1029/2004JA010401.

Jordanova, V. K. (2006), Modeling the behavior of corotating interaction region driven storms in comparison with coronal mass ejection driven storms, in Recurrent Magnetic Storms: Corotating Solar Wind, Geophys. Monogr. Ser., vol. 167, edited by R. McPherron et al., p. 77, AGU, Washington, D. C.

Jordanova, V. K., L. M. Kistler, J. U. Kozyra, G. V. Khazanov, and A. F. Nagy (1996), Collisional losses of ring current ions, J. Geophys. Res., 101,111 .

Jordanova, V. K., J. U. Kozyra, A. F. Nagy, and G. V. Khazanov (1997), Kinetic model of the ring current-atmosphere interactions, J. Geophys. Res., 102, 14,279.

Jordanova, V. K., Y. S. Miyoshi, S. Zaharia, M. F. Thomsen, G. D. Reeves, D. S. Evans, C. G. Mouikis, and J. F. Fennell (2006), Kinetic simulations of ring current evolution during the Geospace Environment Modeling challenge events, J. Geophys. Res., 111, A11S10, doi:10.1029/ 2006JA011644

Jordanova, V. K., S. Zaharia, and D. T. Welling (2010), Comparative study of ring current development using empirical, dipolar, and self-consistent magnetic field simulations, J. Geophys. Res., 115, A00J11, doi:10.1029/ 2010JA015671.

Lavraud, B., V. K. Jordanova, and M. F. Thomsen (2008), Modeling the effects of local time variation of plasma sheet properties on proton ring current energy and peak location, J. Geophys. Res., 113, A05215, doi:10.1029/2007JA012883.

Lennartsson, W., and E. G. Shelley (1986), Survey of 0.1- to $16-\mathrm{keV} / \mathrm{e}$ plasma sheet ion composition, J. Geophys. Res., 91, 3061.

Lu, G., et al. (1994), Interhemispheric asymmetry of the high-latitude ionospheric convection pattern, J. Geophys. Res., 99, 6491.

Lyon, J., J. Fedder, and C. Mobarry (2004), The Lyon-Fedder-Mobarry (LFM) global MHD magnetospheric simulation code, J. Atmos. Sol. Terr. Phys., 66, 1333.

Ma, Y., A. F. Nagy, K. C. Hansen, D. L. De Zeeuw, T. I. Gombosi, and K. G. Powell (2002), Three-dimensional multispecies MHD studies of the solar wind interaction with Mars in the presence of crustal fields, J. Geophys. Res., 107(A10), 1282, doi:10.1029/2002JA009293.

McComas, D. J., S. J. Bame, B. L. Barraclough, J. R. Donart, R. C. Elphic, J. T. Gosling, M. B. Moldwin, K. R. Moore, and M. F. Thomsen (1993), Magnetospheric plasma analyzer: Initial three-spacecraft observations from geosynchronous orbit, J. Geophys. Res., 98, 13,453.

Nosé, M., R. W. McEntire, and S. P. Christon (2003), Change of the plasma sheet ion composition during magnetic storm development observed by the Geotail spacecraft, J. Geophys. Res., 108(A5), 1201, doi:10.1029/2002JA009660.

Ohtani, S., M. Nosé, G. Rostoker, H. Singer, A. T. Y. Lui, and M. Nakamura (2001), Storm-substorm relationship: Contribution of the tail current to Dst, J. Geophys. Res., 106, 21,199.

Powell, K., P. Roe, T. Linde, T. Gombosi, and D. L. De Zeeuw (1999), A solution-adaptive upwind scheme for ideal magnetohydrodynamics, J. Comput. Phys., 154, 284.

Ridley, A. J., and E. Kihn (2004), Polar cap index comparisons with AMIE cross polar cap potential, electric field, and polar cap area, Geophys. Res. Lett., 31, L07801, doi:10.1029/2003GL019113.

Ridley, A. J., and M. W. Liemohn (2002), A model-derived storm time asymmetric ring current driven electric field description, J. Geophys. Res., 107(A8), 1151, doi:10.1029/2001JA000051.

Ridley, A. J., K. C. Hansen, G. Tóth, D. L. De Zueew, T. I. Gombosi, and K. G. Powell (2002), University of Michigan MHD results of the Geospace Global Circulation Model metrics challenge, J. Geophys. Res., 107(A10), 1290, doi:10.1029/2001JA000253.

Ridley, A. J., T. I. Gombosi, and D. L. De Zeeuw (2004), Ionospheric control of the magnetospheric configuration: Conductance, Ann. Geophys., 22,567

Schunk, R. W., J. J. Sojka, and M. D. Bowline (1986), Theoretical study of the electron temperature in the high-latitude ionosphere for solar maximum and winter conditions, J. Geophys. Res., 91, 12,041, doi:10.1029/ JA091iA11p12041

Sckopke, N. (1966), A general relation between the energy of trapped particles and the disturbance field over the Earth, J. Geophys. Res., 71, 3125.

Sharp, R. D., W. Lennartsson, and R. J. Strangeway (1985), The ionospheric contribution to the plasma environment in near-Earth space, Radio Sci., 20, 456, doi:10.1029/RS020i003p00456.

Shelley, E. G., R. G. Johnson, and R. D. Sharp (1974), Morphology of energetic $\mathrm{O}^{+}$in the magnetosphere, in Magnetospheric Physics, Astrophys. Space Sci. Libr., vol. 44, edited by B. M. McCormac, p. 135, D. Reidel, Dordrecht, Netherlands.

Strangeway, R. J., R. E. Ergun, Y. J. Su, C. W. Carlson, and R. C. Elphic (2005), Factors controlling ionospheric outflows as observed at intermediate altitudes, J. Geophys. Res., 110, A03221, doi:10.1029/2004JA010829.

Tóth, G., et al. (2005), Space Weather Modeling Framework: A new tool for the space science community, J. Geophys. Res., 110, A12226, doi:10.1029/2005JA011126.

Tóth, G., D. L. De Zeeuw, T. I. Gombosi, W. B. Manchester, A. J. Ridley, I. V. Sokolov, and I. I. Roussev (2007), Sun-to-thermosphere simulation of the 28-30 October 2003 storm with the Space Weather Modeling Framework, Space Weather, 5, S06003, doi:10.1029/2006SW000272.

Turner, N. E., and D. N. Baker (2000), Evaluation of the tail current contribution to Dst, J. Geophys. Res., 105, 5431.

Walker, R. J., R. L. Richard, T. Ogino, and M. Ashour-Abdalla (1998), The response of the magnetotail to changes in the IMF orientation: The magnetotail's long memory, Phys. Chem. Earth, 24(1-3), 221.

Wang, H., A. J. Ridley, and H. Lühr (2008), Validation of the Space Weather Modeling Framework using observations from CHAMP and DMSP, Space Weather, 6, S03001, doi:10.1029/2007SW000355.

Weimer, D. (1995), Models of high-latitude electric potentials derived with a least error fit of spherical harmonic coefficients, J. Geophys. Res., 100, 19,595 . 
Welling, D. T., and A. J. Ridley (2010a), Validation of SWMF magnetic field and plasma, Space Weather, 8, S03002, doi:10.1029/2009SW000494.

Welling, D. T., and A. J. Ridley (2010b), Exploring sources of magnetospheric plasma using multispecies MHD, J. Geophys. Res., 115 A04201, doi:10.1029/2009JA014596.

Wiltberger, M., W. Lotko, J. G. Lyon, P. Damiano, and V. Merkin (2010), Influence of cusp $\mathrm{O}^{+}$outflow on magnetotail dynamics in a multifluid MHD model of the magnetosphere, J. Geophys. Res., 115, A00J05, doi:10.1029/2010JA015579.

Winglee, R. M. (1998), Multi-fluid simulations of the magnetosphere: The identification of the geopause and its variation with IMF, Geophys. Res. Lett., 25, 4441.

Winglee, R. M., D. Chua, M. Brittnacher, G. K. Parks, and G. Lu (2002), Global impact of ionospheric outflows on the dynamics of the magnetosphere and cross-polar cap potential, J. Geophys. Res., 107(A9), 1237, doi:10.1029/2001JA000214

Yau, A. W., T. Abe, and W. K. Peterson (2007), The polar wind: Recent observations, J. Atmos. Sol. Terr. Phys., 69, 1936, doi:10.1016/j. jastp.2007.08.010

Young, D. T., H. Balsiger, and J. Geiss (1982), Correlations of magnetospheric ion composition with geomagnetic and solar activity, J. Geophys. Res., 87, 9077.

Yu, Y., and A. J. Ridley (2008), Validation of the Space Weather Modeling Framework using ground-based magnetometers, Space Weather, 6, S05002, doi:10.1029/2007SW000345.

Zaharia, S. (2008), Improved Euler potential method for three-dimensional magnetospheric equilibrium, J. Geophys. Res., 113, A08221, doi:10.1029/ 2008JA013325.
Zaharia, S., C. Z. Cheng, and K. Maezawa (2004), 3-D force-balanced magnetospheric configurations, Ann. Geophys., 22, 251.

Zaharia, S., M. F. Thomsen, J. Birn, M. H. Denton, V. K. Jordanova, and C. Z. Cheng (2005), Effect of storm-time plasma pressure on the magnetic field in the inner magnetosphere, Geophys. Res. Lett., 32, L03102, doi:10.1029/2004GL021491.

Zaharia, S., V. K. Jordanova, M. F. Thomsen, and G. D. Reeves (2006), Self-consistent modeling of magnetic fields and plasmas in the inner magnetosphere: Application to a geomagnetic storm, J. Geophys. Res., 111, A11S14, doi:10.1029/2006JA011619.

Zaharia, S., V. K. Jordanova, D. Welling, and G. Tóth (2010), Self-consistent inner magnetosphere simulation driven by a global MHD model, $J$. Geophys. Res., 115, A12228, doi:10.1029/2010JA015915.

Zhang, J., et al. (2007), Understanding storm-time ring current development through data-model comparisons of a moderate storm, J. Geophys. Res., 112, A04208, doi:10.1029/2006JA011846.

Zheng, Y., T. E. Moore, F. S. Mozer, C. T. Russell, and R. J. Strangeway (2005), Polar study of ionospheric ion outflow versus energy input, J. Geophys. Res., 110, A07210, doi:10.1029/2004JA010995.

A. Glocer, NASA Goddard Space Flight Center, 8800 Greenbelt Rd., Greenbelt, MD 20771, USA. (alex.glocer-1@nasa.gov)

V. K. Jordanova, D. T. Welling, and S. G. Zaharia, Los Alamos National Laboratory, PO Box 1663, Los Alamos, NM 87545, USA. (vania@lanl. gov; dwelling@lanl.gov; szaharia@lanl.gov)

G. Toth, Department of Atmospheric, Oceanic and Space Sciences, 2455 Hayward St., University of Michigan, Ann Arbor, MI 48109-2143, USA. (gtoth@umich.edu) 